\title{
Um Dashboard para Análise de Indicadores de Continuidade relacionados à Interrupções no Fornecimento de Energia Elétrica por Causas Ambientais
}

\author{
Márcio Azevedo Rocha ${ }^{1}$, Marcílio Ferreira de Souza Júnior ${ }^{2}$ \\ ${ }^{1}$ Programa de Pós-graduação em Tecnologias Ambientais (PPGTEC) - Instituto Federal \\ de Alagoas (IFAL) \\ Marechal Deodoro - AL - Brasil \\ ${ }^{2}$ CODAI - Universidade Federal Rural de Pernambuco (UFRPE) \\ São Lourenço da Mata - PE - Brasil \\ marcio.rochadifal.edu.br, marcilio.souzajreufrpe.br
}

\begin{abstract}
This research aimed to implement a dashboard based on business intelligence for the analysis of continuity indicators related to the interruption in the electric energy supply due to environmental causes. Dashboard was integrated with legacy databases with the history of power failures and interruptions recorded from 2013 to 2018. It was possible to identify that the environmental causes corresponded to $19.31 \%$ of the concessionaire's equivalent frequency of interruption (FEC) and $23.36 \%$ of the equivalent duration (DEC), revealing that $1 / 5$ of the problems are related to trees, vegetation, winds and fires.
\end{abstract}

Resumo. Esta pesquisa teve como objetivo implementar um dashboard baseado em business intelligence para análise dos indicadores de continuidade relacionados à interrupção no fornecimento de energia elétrica de uma concessionária por causas ambientais. O dashboard foi integrado aos bancos de dados com o histórico de falhas e interrupções de energia registradas pela concessionária estudada no período de 2013 a 2018. Foi possivel identificar que as causas ambientais corresponderam a 19,31\% da frequência equivalente de interrupção (FEC) e 23,36\% da duração equivalente de interrupção (DEC), revelando que 1/5 dos problemas está relacionada com árvores, vegetação, ventos, queimadas e incêndios.

\section{Introdução}

A gestão do fornecimento de energia elétrica implica em analisar as métricas estabelecidas pela Agência Nacional de Energia Elétrica (ANEEL), como os indicadores de continuidade. Esses indicadores podem ser descritos como uma representação quantificável do desempenho de um sistema elétrico e é utilizado para a mensuração da continuidade apurada do fornecimento de energia, comparando-a aos padrões estabelecidos pela agência reguladora [Bandeira, Arrifano e Corrêa 2014].

Os dois principais indicadores coletivos de continuidade definidos pela ANEEL são o de Duração Equivalente de Interrupção por Unidade Consumidora (DEC), que indica o número de horas em que, na média, as unidades consumidoras de um 
determinado conjunto elétrico ficaram sem energia; e o de Frequência Equivalente de Interrupção por Unidade Consumidora (FEC), que expressa a quantidade de vezes, em média, em que as unidades consumidoras de determinado conjunto elétrico sofreram uma interrupção no fornecimento de energia [Tanure 2004; ANEEL 2016].

Desta forma, o desempenho de uma rede de distribuição de energia elétrica pode ser avaliado por meio do histórico de interrupções e da queda de tensão na rede. No caso de violação dos padrões dos indicadores de continuidade em determinado período de apuração, a concessionária pode sofrer advertências e até multas [Borges e Borges, 2014; ANEEL 2016].

É comum a constatação de falhas na rede elétrica oriundas de causas ambientais, como aquelas relacionadas à queda de árvores e contatos com a vegetação, que interferem na qualidade do serviço prestado pelas concessionárias [Cebrian e Kagan, 2010; MME 2016]. As avaliações do desempenho da rede devido às interrupções por causas ambientais podem se tornar gerencialmente complexas por parte das concessionárias de energia à medida que várias informações sobre manutenção preventiva e corretiva precisam ser visualizadas para tomada de decisão [Goldemberg e Lucon 2008].

Neste cenário de tomada de decisão, as soluções de Inteligência de Negócio ou Business Intelligence (BI), que possuem o objetivo de sistematizar os processos de coleta, padronizacão, medição e análise de indicadores de gestão [Radenković et al. 2018], podem fornecer suporte aos gestores por disponibilizar dashboards que incorporam características visuais e funcionais para interpretação de informações [Isik, Jones e Sidorova 2013; Carvalho e Melo 2018; Almeida et al. 2019]. Negash (2004) define BI como um sistema que combina coleta, armazenamento de dados e gerenciamento de conhecimento com ferramentas analíticas para apresentar informações complexas. Para Turban e Volonimo (2013), BI envolve capturar, acessar, entender, analisar e transformar dados brutos em informações estratégicas para melhorar $\mathrm{o}$ desempenho corporativo.

Face à problemática de interrupção de energia elétrica, esta pesquisa teve como objetivo caracterizar as causas relacionadas ao meio ambiente das ocorrências por interrupções no fornecimento de energia elétrica de uma concessionária, a fím de implementar um dashboard baseado em inteligência de negócios para análise dos indicadores de continuidade. O dashboard foi integrado aos bancos de dados com o histórico das ocorrências de falhas e interrupções de energia registradas pela concessionária estudada, compreendendo o período de 2013 a 2018. Com base neste histórico, o dashboard permitiu a visualização segregada dos indicadores DEC e FEC por um grupo focal, composto por gestores da concessionária, visando analisar as causas ambientais que afetaram o fornecimento de energia, como: árvore, vegetação, ventos, queimadas e incêndios, erosões, animais e descargas atmosféricas na rede elétrica.

O restante deste artigo está organizado do seguinte modo: a seção 2 discute os trabalhos correlatos utilizados para fins de comparação com o presente estudo; a seção 3 explana os procedimentos metodológicos da pesquisa; a seção 4 apresenta os resultados alcançados com a criação do dashboard com indicadores para o setor elétrico focado em causas ambientais; por fim, a seção 5 apresenta as considerações finais do estudo. 


\section{Trabalhos correlatos}

Esta seção apresenta alguns trabalhos relacionados a presente pesquisa para fins de comparação dos resultados alcançados. Para tanto, foi empreendida uma pesquisa bibliográfica ad-hoc, ou seja, uma busca foi realizada de forma livre nas fontes, sem a utilização de um protocolo sistemático. Inicialmente, buscou-se trabalhos correlatos ao estudo no portal SBC OpenLib (disponível em https://sol.sbc.org.br/index.php/indice). Em seguida, a busca utilizou como fonte o Google Scholar usando as seguintes palavras-chaves: "dashboard", "setor elétrico", "indicadores" e "meio ambiente". Quatro estudos considerados relevantes foram identificados e são discutidos a seguir.

Em Portugal, Sanz (2018) propôs um dashboard para monitorar falhas de energia comunicando-se com os contadores inteligentes instalados na rede elétrica. A partir de entrevistas com especialistas do setor, foram definidas métricas e indicadores de desempenho para visualização no dashboard de modo adequado aos objetivos da empresa portuguesa. Contudo, o contexto do estudo possui outro tipo de regulação governamental e o foco não eram as causas ambientais de interrupção do fornecimento de energia.

No contexto brasileiro, Silva (2012) adotou uma ferramenta de BI para elaborar relatórios e dashboards com indicadores de perturbação e desligamento forçado na rede elétrica. O trabalho atendeu ao propósito de servir como apoio à tomada de decisões dos gestores, sendo capaz de permitir análises de ocorrências de perturbações no sistema elétrica e foi capaz de processar um grande volume de dados. Contudo, os indicadores trabalhados não foram os regulamentados pela ANEEL e não houve foco nas interrupções ocasionadas por fatores ambientais.

Carrara (2018) usou o Microsoft Excel ${ }^{\odot}$ para desenvolver um dashboard para análise da qualidade do serviço prestado pelas concessionárias de distribuição de energia elétrica, englobando cinco indicadores de desempenho, além do DEC e FEC. Mais uma vez o foco não foram as causas ambientais que poderiam impactar negativamente nos indicadores. Ademais, a solução usando planilha para criar o dashboard não oferece uma gama de recursos necessários para análises de diversas variáveis em tempo real e online. Já Gonçalves (2019) implementou uma abordagem com BI para priorização de obras de uma concessionária, criando dashboards interativos que substituíram planilhas, entretanto, as questões ambientais que poderiam perturbar a rede elétrica também não foram tratadas, tal como proposto pelo presente estudo.

\section{Metodologia da Pesquisa e Arquitetura do BI}

A pesquisa se ambientou em uma concessionária de distribuição de energia elétrica, sediada no Nordeste, que é responsável por atender aproximadamente 1,13 milhões de unidades consumidoras nos municípios sob concessão, possuindo, atualmente, índices de continuidade DEC e FEC que ultrapassam o limite estipulado pela ANEEL.

Os dados transacionais registrados nos sistemas de informação da concessionária foram cedidos a partir da assinatura de um termo de cooperação técnica, compreendendo o período de 2013 a 2018, que totalizou 263.168 registros de ocorrências. Conforme ilustrado na figura 1 , os dados sobre as ocorrências por interrupção são cadastrados a partir de reclamações dos clientes via telefonema 0800 por falta de energia (Sistema 
OPER). Esses dados são associados a outra base com informações técnicas sobre o código da unidade consumidora, tipo de ligação, faturas e adimplência dos clientes (Sistema SGIO). Por último, o sistema de gerenciamento dos ativos e rede elétrica (Sistema SGD) armazena os dados sobre a estrutura elétrica conectada à localização do cliente que gerou a ocorrência. A partir desses três sistemas são gerados os relatórios emitidos para ANEEL, que compõem os indicadores DEC e FEC da concessionária.

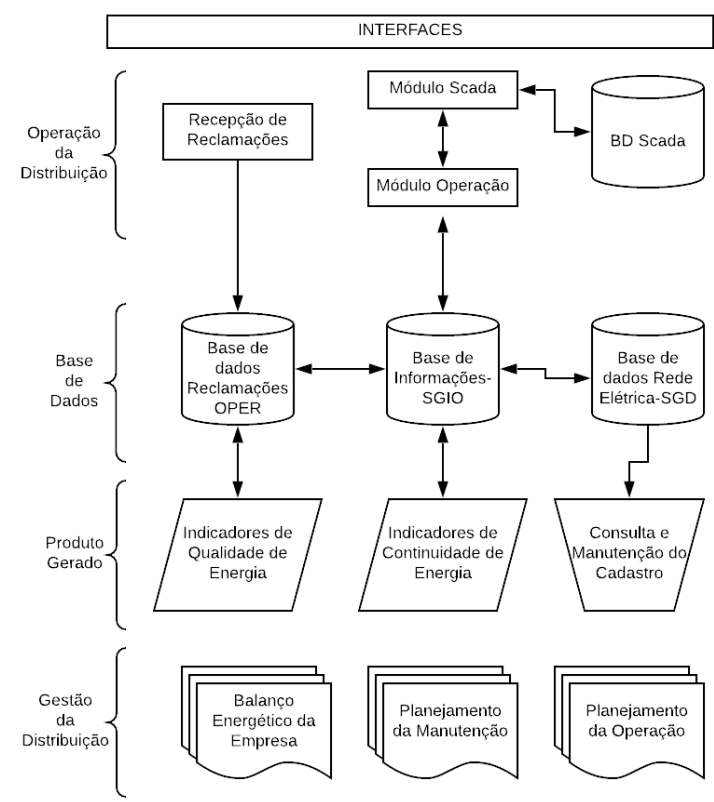

\section{Figura 1 - Bancos de dados históricos da concessionária que forneceram os dados transacionais para a criação do dashboard da pesquisa}

O delineamento da pesquisa também envolveu a realização de um grupo focal com a participação de 4 (quatro) gestores da concessionária para compreender as percepções a respeito dos indicadores de continuidade regulados pela ANEEL e os impactos negativos das falhas por causas ambientais. Esses participantes foram selecionados por ocuparem cargos de liderança dentro da empresa e serem os responsáveis diretos pelo processo de tomada de decisão no setor de manutenção na rede elétrica de distribuição. Foram realizadas duas sessões com o grupo focal, com duração total de 03 horas, de modo a apresentar a proposta e a metodologia de desenvolvimento do ambiente de BI e dos indicadores propostos.

Portanto, as informações consideradas importantes para os gestores foram mapeadas nas bases de dados da concessionária. $\mathrm{O}$ tratamento das três bases legadas definiu a arquitetura e o projeto da aplicação do BI, sendo definidos os atributos e as dimensões do modelo multidimensional [Kimball e Ross 2013], com seus devidos relacionamentos (figura 2 ). 


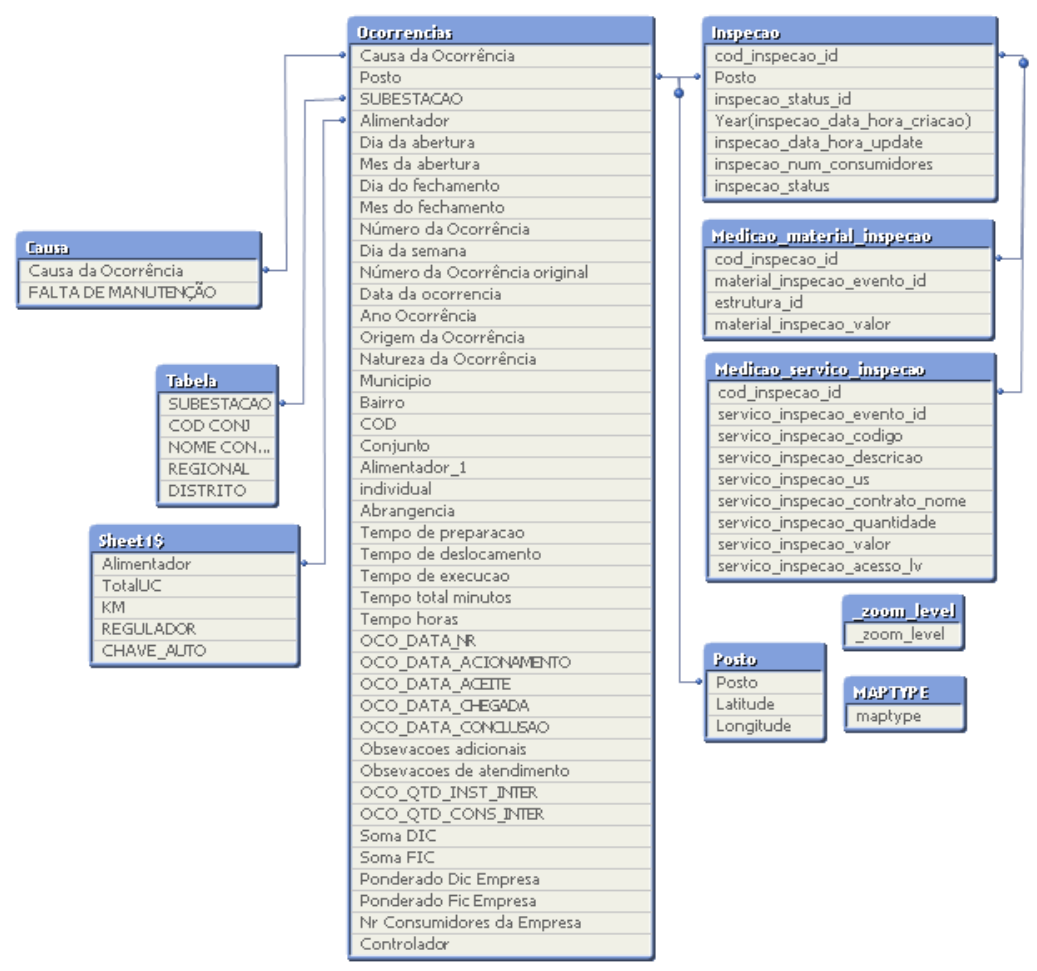

Figura 2 - Dimensões e atributos do BI com seus relacionamentos

Conforme ilustrado acima, os dados do BI foram organizados em 8 (oito) dimensões, que foram: ocorrências, com objetivo de armazenar os dados das reclamações dos clientes (causa da ocorrência, dia da abertura, mês da abertura, dia e mês do fechamento, data, ano e origem da ocorrência); causas, que armazena os dados das causas da ocorrência por falta ou não de manutenção; subestação, que armazena os dados da subestação elétrica, conjunto, regional e distrito da unidade consumidora; alimentadores, que registra os dados dos alimentadores e localização da rede, assim como a quantidade de clientes atendidos; inspeção, que registra os dados dos postos de inspeção e a situação da rede; medicao_material_inspecao, que salva os materiais usados na inspeção; medicao_servico_inspecao, que armazena o tipo do serviço realizado e o custo total dos insumos; posto, onde constam os postos de atendimento da empresa visando a logística de deslocamento das equipes técnicas de campo.

Para o desenvolvimento do BI e criação do dashboard foi adotada a ferramenta QlikView versão 11.0. Os dados históricos foram convertidos através de um processo de limpeza, tratamento e classificação (ETL), sendo carregados para uma área temporária de armazenamento e de transição (Data Stage Area) para manter a qualidade, controle e cruzamento desses dados. Em seguida, os dados já higienizados, foram carregados no QlikView, cujas associações foram executadas através da linguagem AQL (Associative Query Logic) e transferidos ao banco de dados do Data Warehouse (DW) da pesquisa. O DW foi sedimentando em dados menores e associativos, formando uma coleção de dados relacionados a alguma área ou departamento da concessionária. Aplicando o cubo OLAP foi possível executar o pivoteamento com inversão dos dados para dimensões diferentes de visualização, onde pôde-se cruzar as informações e transformá-las em novos padrões, aprofundando os níveis de hierarquia de dados das três principais 
dimensões (ocorrências-OCO, medições-MED e inspeções-INSP), o que aumentou a velocidade de processamento analítico dos dados, inclusive sendo capaz de permitir o acesso offline aos dados. Todas estas etapas encontram-se ilustradas na figura 3.

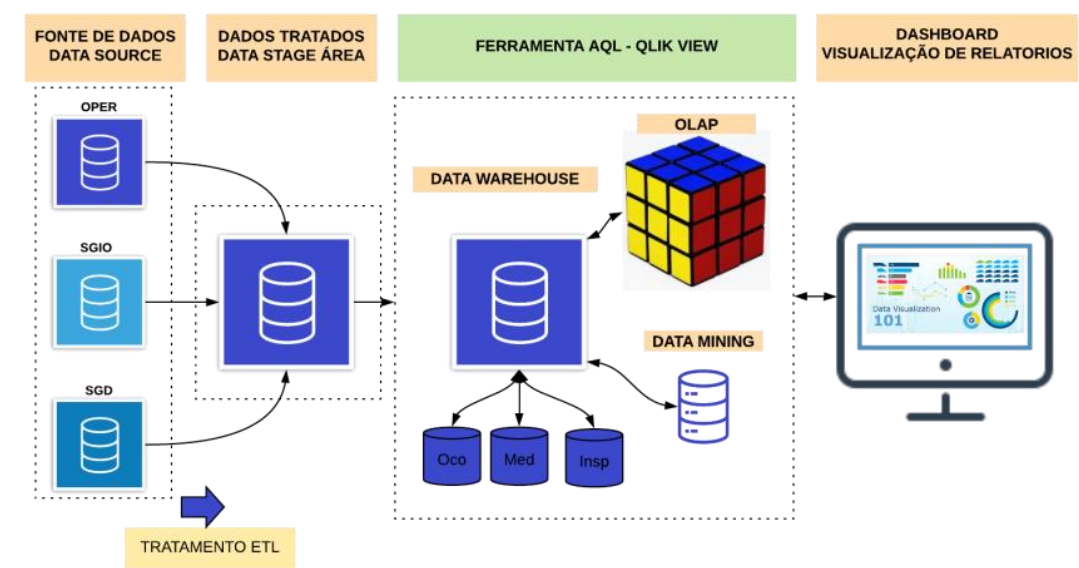

Figura 3- Arquitetura do $\mathrm{BI}$ com os indicadores de continuidade da concessionária de energia elétrica

\section{Dashboard com indicadores de continuidade no fornecimento de energia elétrica relacionados a causas ambientais}

O ambiente do dashboard foi concebido a partir da necessidade de mensurar o impacto do meio ambiente nas ocorrências de interrupção no fornecimento de energia elétrica, visando minimizá-las estrategicamente, além de cruzar as informações de modo que se possa mitigar as métricas capazes de auxiliar à tomada de decisão dos gestores da concessionária tendo em vista a regulação da ANEEL.

A primeira tela do dashboard (figura 4) apresenta uma visão geral sobre os índices operacionais, contendo dados sobre percentual de DEC e FEC por região (leste, metropolitana e oeste) e principais causas de ocorrência. Pôde-se constatar que a causa ambiental de "Ambiente-Árvore/Vegetação" atingiu 9,30\% de FEC e 11,99\% de DEC, considerados valores altos (o ideal seriam valores abaixo de 5\%, segundo a ANEEL) e que necessitam de uma ação mais efetiva de manutenção por parte da concessionária.

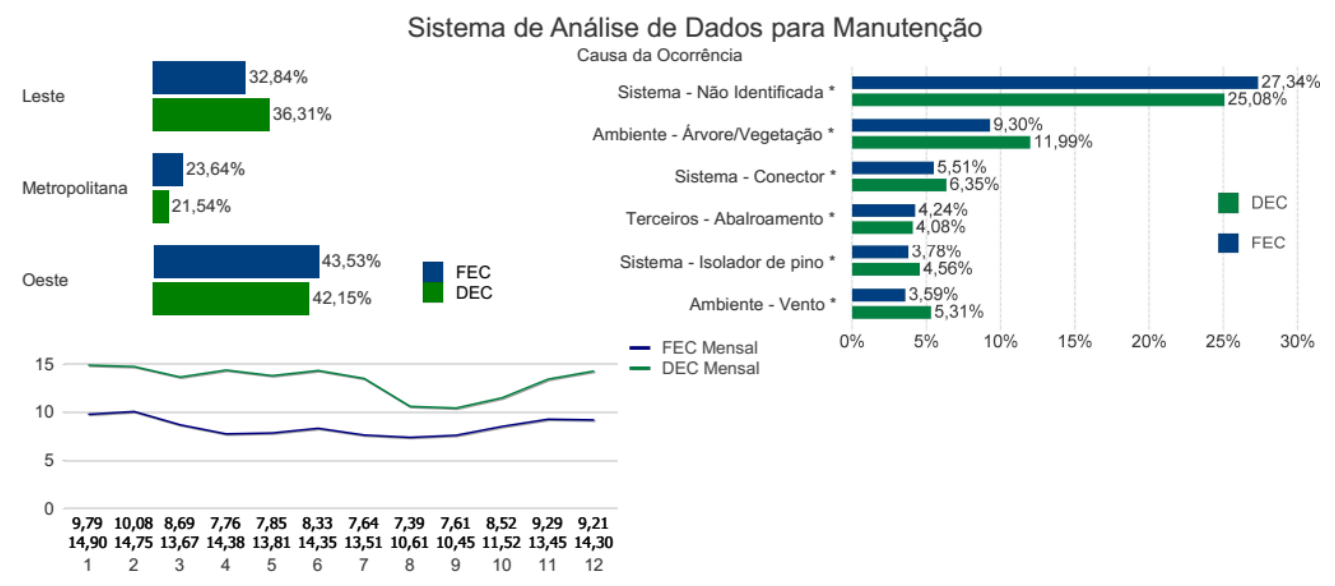

Figura 4 - Dashboard com uma visão geral dos índices no período de 5 anos 
Já a tela do indicador FEC-RESUMO ilustrada no dashboard (figura 5) teve como objetivo visualizar os dados estratificados, para que se obtivesse um afunilamento contínuo dos problemas até se chegar às causas principais das interrupções. Além disso, um mapa de calor indicou aos gestores a região com maiores índices de FEC, possibilitando um planejamento mais adequado.

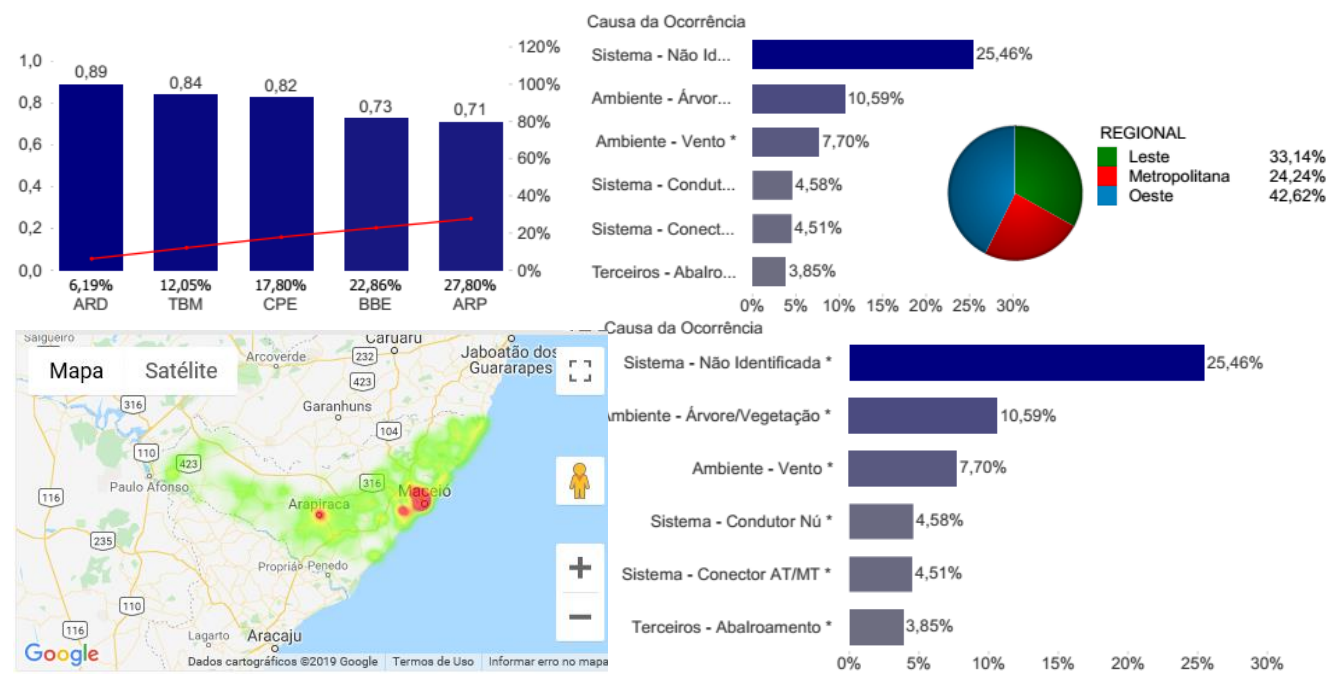

Figura 5. Dashboard com resumo do indicador FEC da concessionária

A partir da tela FEC-RESUMO acima foram aplicados novos filtros para analisar quais os principais locais de influência das causas ambientais, conforme ilustrado na figura 6 .

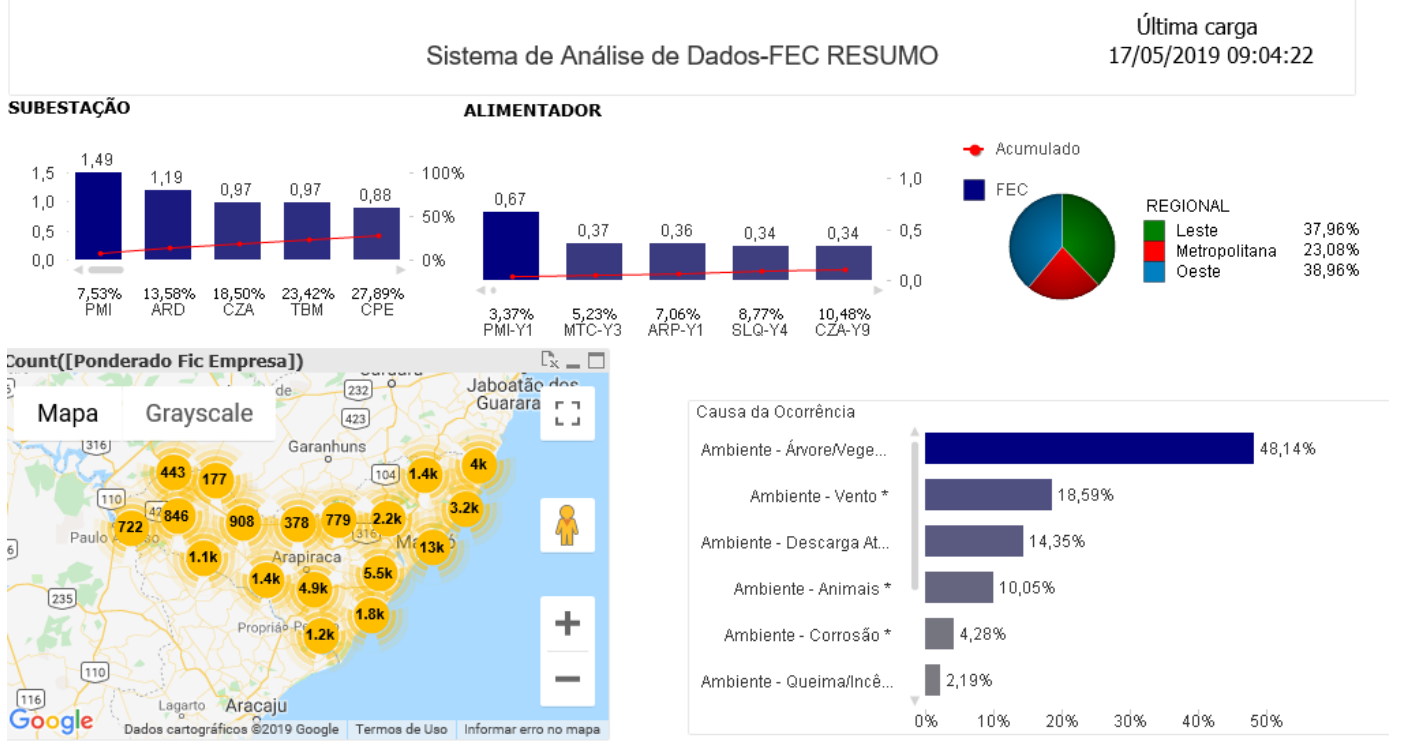

Figura 6 - Dashboard com resumo do indicador FEC relacionado às falhas por causas ambientais

Foi possível constatar que as causas ambientais corresponderam a 19,31\% do FEC e $23,36 \%$ do DEC, sendo que árvores na rede correspondem a maior taxa entre os 
tipos de falhas (figura 6). Notou-se que a subestação "PMI" possuía o maior índice de FEC por falha ambiental durante o período analisado, com $7,53 \%$ de impacto para toda a área de concessão. Esta informação detalhada possibilitaria uma intervenção de melhoria da concessionária neste equipamento, especificamente. Por sua vez, os gráficos de barras na figura representaram o valor absoluto do indicador FEC, enquanto as linhas em vermelho expuseram a participação acumulada de cada conjunto elétrico na mensuração do indicador. O mesmo princípio é utilizado no gráfico por alimentador. Desta forma, foi possível perceber que $48,14 \%$ das causas das ocorrências tinham relação com "Árvore e vegetação" na rede, ocasionando falhas no fornecimento. Ainda, $18,59 \%$ das ocorrências eram relacionadas ao vento, $14,35 \%$ à descarga atmosférica e $10,05 \%$ aos animais. Um mapa de calor presente no dashboard apontou as localizações geográficas onde os problemas foram percebidos e o quantitativo de ocorrências por município da região atendida.

A tabela 1 resume o percentual das causas ambientais caracterizadas pela pesquisa. No que tange os problemas por fatores ambientais, foi possível constatar que correspondem a $1 / 5$ de todas as causas de interrupção no fornecimento de energia elétrica enfrentadas pela concessionária, sendo a falha "Ambiente-Árvore/Vegetação" com maior percentual para cada indicador de continuidade.

\section{Tabela 1 - Principais causas ambientais de interrupção de fornecimento de energia elétrica na concessionária}

\begin{tabular}{|l|l|l|}
\hline \multicolumn{1}{|c|}{ Causa da Ocorrência } & FEC & \multicolumn{1}{c|}{ DEC } \\
\hline Ambiente - Árvore/Vegetação & $48,14 \%$ & $51,34 \%$ \\
\hline Ambiente - Vento & $18,59 \%$ & $22,74 \%$ \\
\hline Ambiente - Descarga Atmosférica & $14,35 \%$ & $12,27 \%$ \\
\hline Ambiente - Animais & $10,05 \%$ & $8,14 \%$ \\
\hline Ambiente - Corrosão & $4,28 \%$ & $3,44 \%$ \\
\hline Ambiente - Queimada & $2,19 \%$ & $0,55 \%$ \\
\hline Ambiente - Incêndio & $1,58 \%$ & $0,78 \%$ \\
\hline Ambiente - Erosão & $0,48 \%$ & $0,43 \%$ \\
\hline Ambiente - Poluição & $0,29 \%$ & $0,23 \%$ \\
\hline Ambiente - Inundação & $0,06 \%$ & $0,08 \%$ \\
\hline
\end{tabular}

Dificuldades como a falta de acompanhamento das equipes técnicas com relação ao crescimento de árvores, ausência de planejamento da arborização nas cidades e implantações na rede do sistema elétrico realizados de forma independente contribuem para o crescimento ainda maior das causas ambientais constatadas pela pesquisa. 


\section{Considerações finais}

A pesquisa teve como objetivo analisar os indicadores de continuidade no fornecimento de energia elétrica relacionados às interrupções por causas ambientais, baseando-se em uma ferramenta computacional de inteligência de negócio. $\mathrm{O}$ caso estudado de uma concessionária nordestina permitiu analisar dados relacionados a 263.168 ocorrências do hitórico da empresa, perfazendo 1,13 milhões de consumidores atendidos na região.

Foi possível identificar que os problemas ambientais corresponderam a 19,31\% do FEC e $23,36 \%$ do DEC da empresa, acarretando que $1 / 5$ dos problemas estão relacionados ao meio ambiente, que deveriam então ser tratados e solucionados com inspeções técnicas. Cabe ressaltar que os valores elevados dos dois indicadores de continuidade significam que as unidades consumidoras de um determinado conjunto elétrico regional ficaram sem energia ou sofreram constantes interrupções no seu fornecimento, o que acarreta no pagamento de multas por parte da concessionária. Sendo assim, o BI desenvolvido torna-se uma ferramenta estratégica importante para os gestores do setor elétrico.

Para trabalhos futuros, propõe-se tratar as informações no BI cruzando os diversos departamentos da concessionária, além do setor de manutenção da rede elétrica. Assim, seria possível identificar: locais com maior adimplência dos clientes para definir atendimentos prioritários; identificação dos locais com mais desvio de energia, criando mapas de calor por região; e cruzamento das localizações com maior consumo de energia a fim de gerenciar mais adequadamente os serviços prestados aos consumidores.

\section{Referências}

Almeida, D., Alves, A., Baptista, C., Figueirêdo, H., Freire, P., Silva, D. (2019) "Uso de Business Intelligence na Gestão de Recursos Hídricos: o caso da Fiscalização do Uso da Água”. In: Anais do X Workshop de Computação Aplicada a Gestão do Meio Ambiente e Recursos Naturais - WCAMA.

ANEEL (2016). Agência Nacional de Energia Elétrica. Procedimentos de Distribuição de Energia Elétrica no Sistema Elétrico Nacional - PRODIST. Disponível em: http://www.aneel.gov.br/documents/656827/14866914/M\%C3\%B3dulo1_Revisao_9 /1b78da82-6503-4965-abc1-a2266eb5f4d7

Bandeira, L. M., Arrifano, R. C. D. e Corrêa, A. (2014). "Análise da Continuidade do Sistema de Distribuição de Energia Elétrica no Estado do Pará". Revista Engenharia Elétrica, v.2, n.2, p.1-12.

Borges, F.Q. e Borges, F.Q. (2014) "Desempenho Institucional e a Gestão da Sustentabilidade: Uma Análise de Indicadores na ANEEL". Revista Economia \& Gestão, v.14, n.35.

Carvalho, R. C. e Melo, C. O. (2018) "Tomada de decisão baseada em dados: avaliando a visualização de informação em dashboards". In: Workshop de Iniciação Científica em Sistemas de Informação - Simpósio Brasileiro de Sistemas de Informação (SBSI), Caxias do Sul.

Carrara, Y. M. (2018) "Desenvolvimento de um Sistema de Análise da Qualidade do Serviço Prestado pelas Concessionárias de Distribuição de Energia Elétrica". TCC 
(Graduação em Engenharia Elétrica) - Faculdade de Engenharia Elétrica, Universidade Federal de Uberlândia.

Cebrian, J. C. e Kagan, N. (2010) "Hybrid Method to Assess Sensitive Process Interruption Costs Due to Faults in Electric Power Distribution Networks". IEEE Transactions on Power Delivery, v.25, n.3, p.1686-1696.

Gonçalves, B. H. M. (2019) "Priorização de Investimentos em Subestações de Alta Tensão com Abordagem Business Intelligence". TCC (Graduação em Engenharia Elétrica) - Departamento de Engenharia Elétrica, Universidade Federal do Paraná.

Goldemberg, J. e Lucon, O. (2008). "Energia, meio ambiente e desenvolvimento". São Paulo: EDUSP.

Isik, O., Jones, M.C. e Sidorova, A. (2013) "Business intelligence success: the roles of BI capabilities and decision environments". Information \& Management, v.5, n.1, p.13-23.

Kimball, R. e Ross, M. (2013). "The Data Warehouse Toolkit: The Definitive Guide to Dimensional Modeling (3. Ed)”. Indianapolis: John Wiley \& Sons, Inc.

Negash, S. (2004) "Business Intelligence". Communications of the Association for Information Systems, v.13, p.177-195.

Radenković, M., Lukić, J., Despotović-Zrakić, M., Labus, A. e Bogdanović, Z. (2018). "Harnessing business intelligence in smart grids: A case of the electricity market". Computers in Industry, v.96, p.40-53.

Sanz, A. C. P. (2018) "Proposta de um dashboard para monitorizar falhas de energia numa rede elétrica inteligente". Dissertação (Mestrado em Gestão de Sistemas de Informação) - Instituto Universitário de Lisboa (ISCTE), Lisboa.

Silva, M. L. (2012) "Modelo de Data Warehouse para a análise de ocorrências de perturbações e desligamentos forçados no Sistema Interligado Nacional". TCC (Graduação em Engenharia Eletrônica e de Computação) - Departamento de Eletrônica e de Computação, Universidade Federal do Rio de Janeiro.

MME (2016). Ministério de Minas e Energia. "Relatório de Avaliação dos Custos Relacionados às Interrupções de Energia Elétrica e suas Implicações na Regulação”. Sinapsis Inovação em Energia http://www.mme.gov.br/documents/36144/472644/Produto+5.pdf

Tanure, J. E. P. S. A. (2004) "Proposta de procedimentos e metodologia para estabelecimento de metas de qualidade (DEC e FEC) para concessionárias de distribuição de energia elétrica através da análise comparativa". Tese (Doutorado em Engenharia Elétrica) - Escola Politécnica, Universidade de São Paulo, São Paulo.

Turban, E. e Volonimo, L. (2013) “Business Intelligence e Suporte à Decisão". Porto Alegre: Bookman. 